\title{
The Prognostic Factors of Preoperative Prognostic Nutritional Index and Radiological Findings of Solid Pseudopapillary Tumors of Pancreas: A Single- Center Experience of 14 Years
}

This article was published in the following Dove Press journal:

Cancer Management and Research

\section{He Song \\ Ming Dong (D)}

Department of Gastrointestinal Surgery of the First Hospital of China Medical University, Shenyang II000I, People's Republic of China
Correspondence: Ming Dong

Tel $+86 \mid 380490065$ I

Fax +86248328288।

Email dongming@cmu.edu.cn
Introduction: Solid pseudopapillary tumors of pancreas (SPTP) is an indolent rare tumor with malignant potential. The prediction of malignancy is an enigma. The aim of this study is to explore the relationship between operative measurements and malignancy prognosis of SPTP patients.

Methods: A cohort of consecutive 102 patients were enrolled in this study. Preoperative measurements and clinical outcomes were analyzed.

Results: Eighteen patients (17.6\%) were confirmed as malignant. The malignant SPTP of the optimal cut-off value was $47.9(\mathrm{p}=0.012)$ for prognostic nutritional index (PNI). The value of $\mathrm{PNI} \leq 47.9$ and incomplete capsule were significantly correlated with malignancy. Univariate analysis showed that the $\mathrm{PNI} \leq 47.9(p=0.013)$ and incomplete capsule $(p<0.001)$ were predictors of disease-specific survival (DSS). Multivariate analysis identified the $\mathrm{PNI} \leq 47.9(p=0.036)$ and incomplete capsule $(p=0.023)$ as the independent prognostic factors of DSS. The new score of $0,1,2$ based on PNI and capsule presence stratified the patients into 3 groups. The patients with low PNI and incomplete capsule achieved the worst prognosis. Conclusion: The combination test of operative PNI and capsule presence would be a reliable indicator of the aggressive natural history of SPTP.

Keywords: solid pseudopapillary tumor, pancreas, prognostic nutritional index, diseasespecific survival

\section{Introduction}

Solid pseudopapillary tumors of pancreas (SPTP) is a low malignant potential tumor predominantly occurring in young women. The majority of SPTP presented with a relatively indolent biological behavior, while others manifested aggressive invasion into adjacent organs or distant metastasis. Papavramids et al and Law et al had, respectively, summed up 718 and 2744 SPTP patients in the English literature. ${ }^{1,2}$ The reports of SPTP have increased markedly since $2000 .^{3}$ Despite the increasing research, the natural history of SPTP is still uncertain. Reliable prognostic variables are essential for clinical management. Some studies indicated risk factors (such as ki67 , microscopic appearance) were related to malignancy and adverse outcomes. ${ }^{4,5}$ However, these variables could only be assessed after surgery. Thus, preoperative findings associated with malignancy behavior should be clarified. 
The progression of malignant tumors is related to the deterioration of the body condition via systemic inflammation and malnutrition. It is well known that nutritional status and cancer-related inflammation could exert a great impact on the pathogenesis of various types of pancreatic tumors. ${ }^{6-9}$ Some inflammatory and nutritional biomarkers, such as the neutrophil to lymphocyte ratio (NLR), platelet-to-lymphocyte ratio (PLR), systemic immune-inflammation index (SII), and the prognostic nutritional index (PNI) are discovered to be widely used to reflect the immunonutritional level. These parameters are simple to perform in regular routine examinations. Nevertheless, few studies were concentrated on the association between the immunonutritional markers and malignancy of SPTP.

In the most widely used imaging examination such as CT (computed tomography), MRI (magnetic resonance imaging), EUS (endoscopic ultrasound), SPTP mainly represents a well-capsulated heterogeneous mass with solid and cystic components. The presence of capsule, the proportion of solid component, and large tumor size may predict malignancy. ${ }^{3,10}$ EUS-FNA (endoscopic ultrasound-fine needle aspiration) is an effective method with extremely high diagnostic rates in SPTP. ${ }^{11}$ However, some researchers regarded EUS-FNA not to be safe enough, and reported that it could cause rupture of tumor and seeding of the needle tract. ${ }^{12}$ EUS-FNA was also an invasive procedure which might influence immunonutritional markers.

The aim of this research was to provide a preoperative scoring method incorporating serum tests and radiological features, which might be an efficient predicting tool for the prognosis in SPTP.

\section{Methods}

\section{Study Design}

This study was a single-center retrospective analysis. From January 2006 to January 2020, 102 consecutive patients with pathologically confirmed SPTP at the Department of General Surgery, the First Hospital of China Medical University were retrospectively reviewed.

The primary inclusion criteria were as follows: 1) age more than 18 years; and 2) complete peripheral serum routine tests and abdominal computed tomography (CT) scans available within a month before surgery. The exclusion criteria were: 1) combined with an infectious disease; 2) having a history of prior malignancy; 3) receiving
ERCP, EUS-FNA or other invasive examination before surgery. Exclusion criteria and the flow chart are shown in Figure 1.

\section{Follow-Up}

All the patients were followed up by telephone interview, outpatient visits, and China's native app, WeChat, and all surviving patients were followed up for 14 years. Patient status was due to the time of the last follow-up as follows: no evidence of disease, alive with disease, dead of disease, dead of other causes. Survival time was determined as starting from the date of the first operation until the end of follow-up due to either death or end of data collection. Only "dead of disease" was considered an event in the analysis of disease-specific survival (DSS).

\section{Data Collection and Clinical Outcome}

Data on patient demographics, preoperative parameters, surgical details, and histopathological results were obtained from medical records.

NLR, PLR, SII, and PNI were calculated using formula: NLR $=$ neutrophil counts/lymphocyte counts; PLR = platelet counts/lymphocyte counts; SII = platelet counts $\times$ neutrophil counts/lymphocyte counts; and PNI

Adult patients (age $\geqslant 18$ ) confirmed as SPTP underwent resection for from January 2006 to January $2020(n=112)$

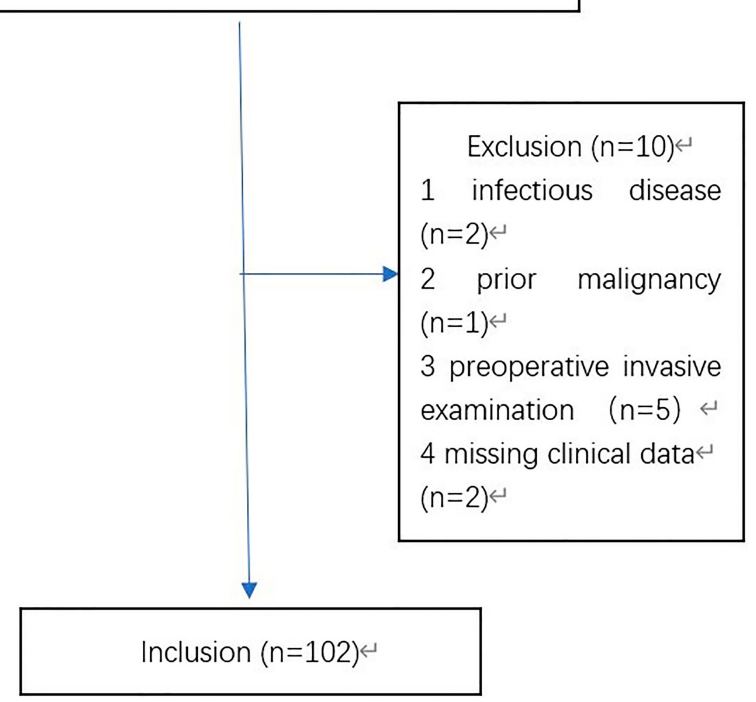

Figure I Flow chart of the SPTP patients in this study. In total, 102 of I 12 patients (91.I\%) could be analyzed after the exclusion of two patients who acquired infectious disease, one patient who had prior lung cancer, five patients who received invasive procedures such as ERCP or EUS-FNA, and two patients who had insufficient clinical data. 


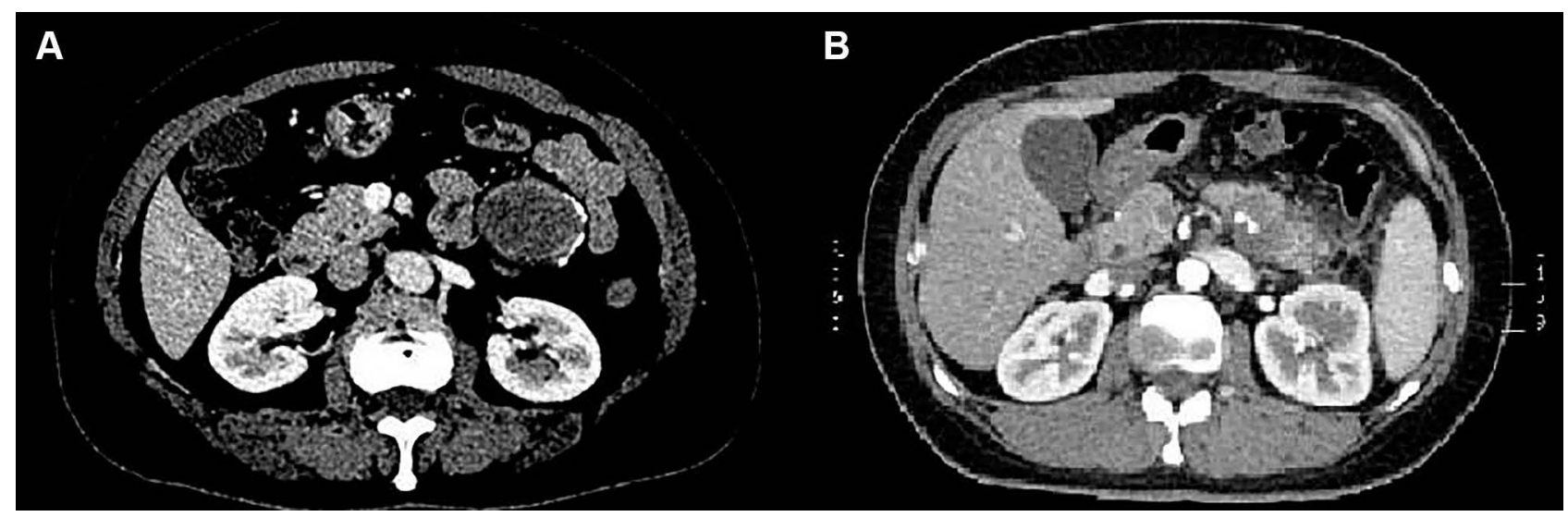

Figure 2 (A) The SPTP located at the tail of pancreas with complete capsule was confirmed to be benign. (B) The SPTP located at the tail of pancreas with incomplete capsule was confirmed to be malignant due to pancreatic parenchymal invasion.

$=$ albumin level $(\mathrm{g} / \mathrm{L})+5 \times$ total lymphocyte counts $\left(10^{9} / \mathrm{L}\right)$.

For the radiological analysis, every image from 102 patients was reviewed with CT or MRI. Each tumor was analyzed according to the following categories: tumor location (head and neck, body and tail, extrapancreatic site), component of the tumor (solid and cystic, solid, cystic), morphology of capsule (complete, incomplete), calcification (present, absent). "Incomplete capsule" was defined as a capsule which does not surround the entire periphery of the SPTP. A typical CT image in our case series including complete and incomplete capsule is shown in Figure 2.

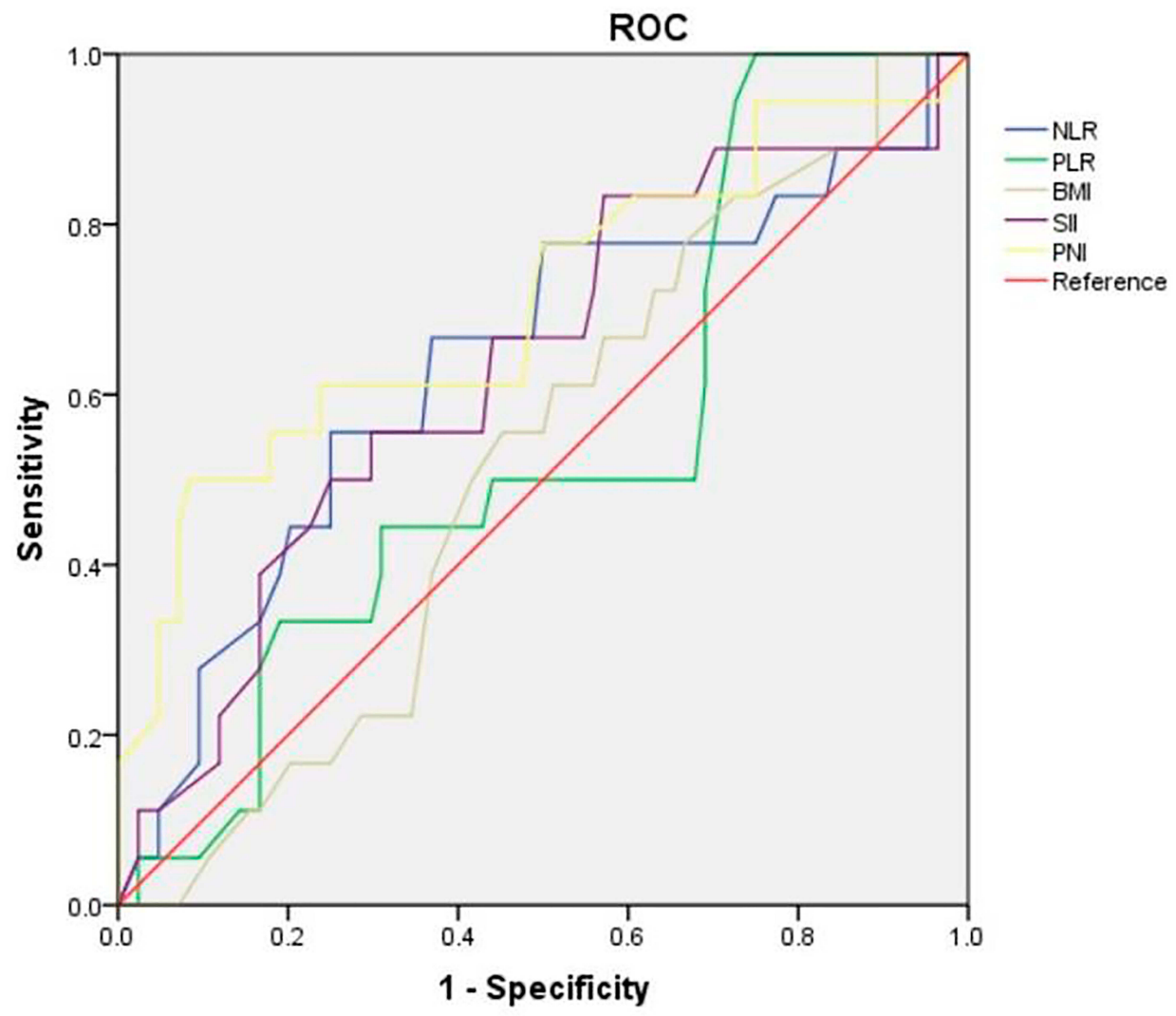

Figure 3 Receiver operating characteristic curves for predicting malignant SPTP by PNI (AUC 0.686), BMI (AUC 0.522), NLR (AUC 0.636), PLR (AUC 0.545), and SII (AUC 0.632). Abbreviation: AUC, area under the curve. 
Table I The Optimal Cutoff of PNI, BMI, NLR, PLR, and SII

\begin{tabular}{|l|l|l|l|}
\hline Parameters & Cutoff & Sensitivity & Specificity \\
\hline PNI & 47.9 & 0.556 & 0.798 \\
BMI & 22.7 & 0.778 & 0.333 \\
NLR & 3.1 & 0.554 & 0.750 \\
PLR & 100.9 & 1 & 0.250 \\
SII & 363.8 & 0.833 & 0.429 \\
\hline
\end{tabular}

Table 2 Clinical Characteristics of SPTP Patients

\begin{tabular}{|c|c|c|}
\hline Parameters & $\begin{array}{l}\text { Patient Number } \\
(n=102)\end{array}$ & $\%$ \\
\hline Age (year, average) & $37.0 \pm 12.8$ & \\
\hline \multicolumn{3}{|l|}{ Gender } \\
\hline Female & 90 & $88.2 \%$ \\
\hline Male & 12 & $11.8 \%$ \\
\hline BMI (average) & $24.3 \pm 2.3$ & \\
\hline \multicolumn{3}{|l|}{ Clinical Presentation } \\
\hline Abdominal pain & 40 & $39.2 \%$ \\
\hline Abdominal distention & 18 & $17.6 \%$ \\
\hline Abdominal mass & 20 & $19.6 \%$ \\
\hline Incidental detection & 49 & $48.0 \%$ \\
\hline Nausea and vomiting & 10 & $9.8 \%$ \\
\hline Back pain & 4 & $3.9 \%$ \\
\hline Hematuria & 2 & $1.8 \%$ \\
\hline \multicolumn{3}{|l|}{ Component of tumor } \\
\hline Solid and cystic & 49 & $48.0 \%$ \\
\hline Solid & 19 & $18.6 \%$ \\
\hline Cystic & 34 & $33.3 \%$ \\
\hline Calcification & 20 & $19.6 \%$ \\
\hline Dilated main pancreatic duct & 3 & $2.9 \%$ \\
\hline Tumor location & & $47.2 \%$ \\
\hline Head+neck & 36 & $35.3 \%$ \\
\hline Body+tail & 65 & $63.7 \%$ \\
\hline Extrapancreatic site & 1 & $0.9 \%$ \\
\hline Tumor size $(\mathrm{cm})$ & $6.6 \pm 3.8 \mathrm{~cm}$ & \\
\hline \multicolumn{3}{|l|}{ Operation type } \\
\hline Pancreaticoduodenectomy & 10 & $9.8 \%$ \\
\hline $\begin{array}{l}\text { Pylorus-preserving } \\
\text { pancreaticoduodenectomy }\end{array}$ & 5 & $4.9 \%$ \\
\hline $\begin{array}{l}\text { Duodenum-preserving pancreatic head } \\
\text { resection }\end{array}$ & 4 & $3.9 \%$ \\
\hline Middle pancreatectomy & 4 & $3.9 \%$ \\
\hline $\begin{array}{l}\text { Distal pancreatectomy with } \\
\text { preserving-spleen }\end{array}$ & 25 & $24.5 \%$ \\
\hline $\begin{array}{l}\text { Distal pancreatectomy with } \\
\text { splenectomy }\end{array}$ & 23 & $22.5 \%$ \\
\hline Enucleation & 29 & $28.4 \%$ \\
\hline Palliative operation & 2 & $1.9 \%$ \\
\hline Surgical period (hour average) & $3.6 \pm 1.1$ & \\
\hline
\end{tabular}

(Continued)
Table 2 (Continued).

\begin{tabular}{|l|l|l|}
\hline Parameters & $\begin{array}{l}\text { Patient Number } \\
\text { (n=102) }\end{array}$ & $\%$ \\
\hline Postsurgical complications & & \\
Pancreatic fistula & 18 & $17.6 \%$ \\
Intra-abdominal abscess & 9 & $8.8 \%$ \\
Gastrointestinal bleeding & 2 & $1.9 \%$ \\
Gastric fistula & 1 & $0.9 \%$ \\
\hline Capsule pattern & & \\
Complete capsule & 85 & $83.4 \%$ \\
Incomplete capsule & 17 & $16.6 \%$ \\
Benign tumor & 84 & $82.3 \%$ \\
Malignant tumor & 18 & $17.6 \%$ \\
Vascular infiltration & 3 & $2.9 \%$ \\
Pancreatic parenchymal invasion & 8 & $7.8 \%$ \\
Adjacent organ invasion & 6 & $5.9 \%$ \\
Perineural invasion & 1 & $0.9 \%$ \\
\hline
\end{tabular}

The definition of malignant SPTP was confirmed by the WHO criteria, such as angioinvasion, perineural invasion, pancreatic parenchymal invasion, or deep infiltration into the surrounding tissue or metastasis.

\section{Statistical Analysis}

Descriptive statistics were represented as mean \pm standard deviation [SD] or as the median (range) or as proportion. The characteristics of the 2 groups (with benign and malignant SPTP) were performed using $\chi^{2}$ test, the Mann-Whitney $U$-test for categorical variables, and independent-sample $t$-test for continuous variables. The optimal cut-off values of continuous variables including NLR, PLR, SII, and PNI for predicting malignant SPTP were determined by using receiver operating characteristic (ROC) curve analysis and the Youden index. The Kaplan-Meier method was used to estimate DSS. Variables with $p<0.1$ in univariable analysis were included in multivariable analysis. A Cox proportionalhazards regression analysis was performed. $A p$-value of less than 0.05 was considered significant. All statistical analyses were performed with the Statistical Package for Social Sciences (SPSS) 24.0 for Windows (Chicago, Illinois).

\section{Results}

\section{Preoperative Examination}

The cohort of 102 cases consisted of 12 male cases and 90 female cases, with a ratio of male/female of 1:7.5. The 
Table 3 Predictive Factors of Malignant SPTP

\begin{tabular}{|c|c|c|c|}
\hline Factor & Benign & Malignant & p-value \\
\hline $\begin{array}{l}\text { Average age } \\
\text { Average BMI } \\
\text { Average NLR } \\
\text { Average PLR } \\
\text { Average SII } \\
\text { Average PNI }\end{array}$ & $\begin{array}{l}36.0 \pm 11.8 \\
23.7 \pm 2.4 \\
2.6 \pm 2.0 \\
151.9 \pm 90.7 \\
654.7 \pm 6 \mid 4.0 \\
51.1 \pm 4.2\end{array}$ & $\begin{array}{l}41.8 \pm 16.6 \\
23.8 \pm 1.9 \\
3.7 \pm 2.6 \\
149.7 \pm 51.7 \\
961.2 \pm 828.4 \\
48.3 \pm 3.8\end{array}$ & $\begin{array}{l}0.084 \\
0.882 \\
0.105 \\
0.918 \\
0.152 \\
0.012\end{array}$ \\
\hline $\begin{array}{l}\text { Gender } \\
\text { Male } \\
\text { Female }\end{array}$ & $\begin{array}{l}10 \\
74\end{array}$ & & 0.924 \\
\hline $\begin{array}{c}\text { Symptoms } \\
\text { Present } \\
\text { Absent }\end{array}$ & $\begin{array}{l}44 \\
40\end{array}$ & $\begin{array}{l}9 \\
9\end{array}$ & 0.854 \\
\hline $\begin{array}{l}\text { Serum tumor marker } \\
\text { Elevated } \\
\text { Normal }\end{array}$ & $\begin{array}{l}7 \\
77\end{array}$ & $\begin{array}{l}1 \\
17\end{array}$ & 0.691 \\
\hline $\begin{array}{l}\text { Average tumor size }(\mathrm{cm}) \\
\qquad 5 \mathrm{~cm} \\
>5 \mathrm{~cm}\end{array}$ & $\begin{array}{l}36 \\
48\end{array}$ & $\begin{array}{l}5 \\
13\end{array}$ & 0.137 \\
\hline $\begin{array}{l}\text { Tumor location } \\
\text { Head+neck } \\
\text { Body and tail } \\
\text { Extrapancreatic site } \\
\text { Calcification condition } \\
\text { Calcification } \\
\text { Non-calcification } \\
\text { Component of tumor } \\
\text { Solid } \\
\text { Solid and cystic } \\
\text { Cystic }\end{array}$ & $\begin{array}{l}27 \\
57 \\
0 \\
16 \\
68 \\
17 \\
43 \\
24\end{array}$ & $\begin{array}{l}9 \\
8 \\
1 \\
4 \\
14 \\
2 \\
6 \\
10\end{array}$ & $\begin{array}{l}0.236 \\
0.749 \\
0.087\end{array}$ \\
\hline $\begin{array}{l}\text { Pattern of capsule } \\
\text { Complete capsule } \\
\text { Incomplete capsule }\end{array}$ & $\begin{array}{l}78 \\
6\end{array}$ & $\begin{array}{l}7 \\
11\end{array}$ & $<0.001$ \\
\hline $\begin{array}{l}\text { PNI } \\
\qquad 47.9 \\
>47.9\end{array}$ & $\begin{array}{l}17 \\
67\end{array}$ & $\begin{array}{l}11 \\
7\end{array}$ & 0.001 \\
\hline
\end{tabular}

average age was $37.0 \pm 12.8$ years (18-71). The clinical presentation of SPTP was non-specific; patients often represent two or more symptoms.

At baseline, the average value of BMI, NLR, PLR, SII, and PNI were, respectively, $24.3 \pm 2.3,2.8 \pm 2.2,151.5 \pm 84.9$, $708.7 \pm 662.6$, and $50.6 \pm 4.3$. Their ranges were 18.5-28.7, 1.1-11.7, 69.5-625.5, 229.9-3721.7, and 42.6-59.6.

The area under curve (AUC) for predicting malignancy of SPTP was $0.686(p=0.012), 0.522(p=0.769), 0.636$ $(p=0.071), 0.545(p=0.551)$ and $0.632(p=0.079)$ for PNI,
BMI, NLR, PLR, and SII (Figure 3). The optimal cut-off PNI, BMI, NLR, PLR, and SII was 47.9, 22.7, 3.1, 100.9, and 363.8 by the highest Youden index (shown in Table 1).

The level of tumor markers, including AFP, CEA, CA199, CA125, and CA242, was slightly increased in 8 patients.

The radiological presentations were consistent with solid and cystic pattern in 49 cases, solid pattern in 19 cases, and cystic pattern in 34 cases. Calcification in the tumor and dilated main pancreatic duct were reported for 20 patients and 3 patients. There were 85 cases completely encapsulated and 17 cases incompletely encapsulated.

There were 36 cases $(35.3 \%)$ distributed on the pancreatic head and neck, 65 cases (63.7\%) distributed on the pancreatic body and tail, and 1 case $(0.9 \%)$ distributed on the extrapancreatic site (located at a site surrounded by the left kidney, spleen, and pancreatic tail). The mean size of the tumors was $6.6 \pm 3.8 \mathrm{~cm}(2-15 \mathrm{~cm})$.

\section{Surgery and Postoperative Outcome}

$\mathrm{R}_{0}$ resection was performed in 100 cases, while a palliative operation was performed in 2 cases. Radical resection was impossible, and cholangiojejunostomy was performed in 2 cases because massive superior mesenteric vein involvement made mass reduction and graft reconstruction unfeasible. Total surgical period was $3.6 \pm 1.1 \mathrm{~h}(1-9.1 \mathrm{~h})$.

No one died during the perioperative period. Postsurgical complications occurred in 30 patients. The most common complication was pancreatic fistula (18 cases), followed by intra-abdominal abscess ( 9 cases), gastrointestinal bleeding ( 2 cases), gastric fistula (1 case). These complications were resolved by conservative therapy except for one case undergoing second surgery for gastrointestinal bleeding.

Immunohistochemical staining showed that alpha 1antichymotrypsin (AACT), vimentin, neuron-specific enolase (NSE), ki-67 appearing positive expression mostly and the positive rates for them were 95.9\% (95/99), $92.7 \% \quad(90 / 97), \quad 75 \% \quad(60 / 80), \quad$ and $71.1 \% \quad(32 / 45)$ respectively.

Statistics for all the above are listed in Table 2.

\section{Characteristics of Malignant SPTP}

All the cases (102 cases) were pathologically confirmed as SPTP, and 18 cases $(17.6 \%)$ were diagnosed as malignant, as follows: vascular infiltration was identified in 3 patients, pancreatic parenchymal invasion concurrent with 


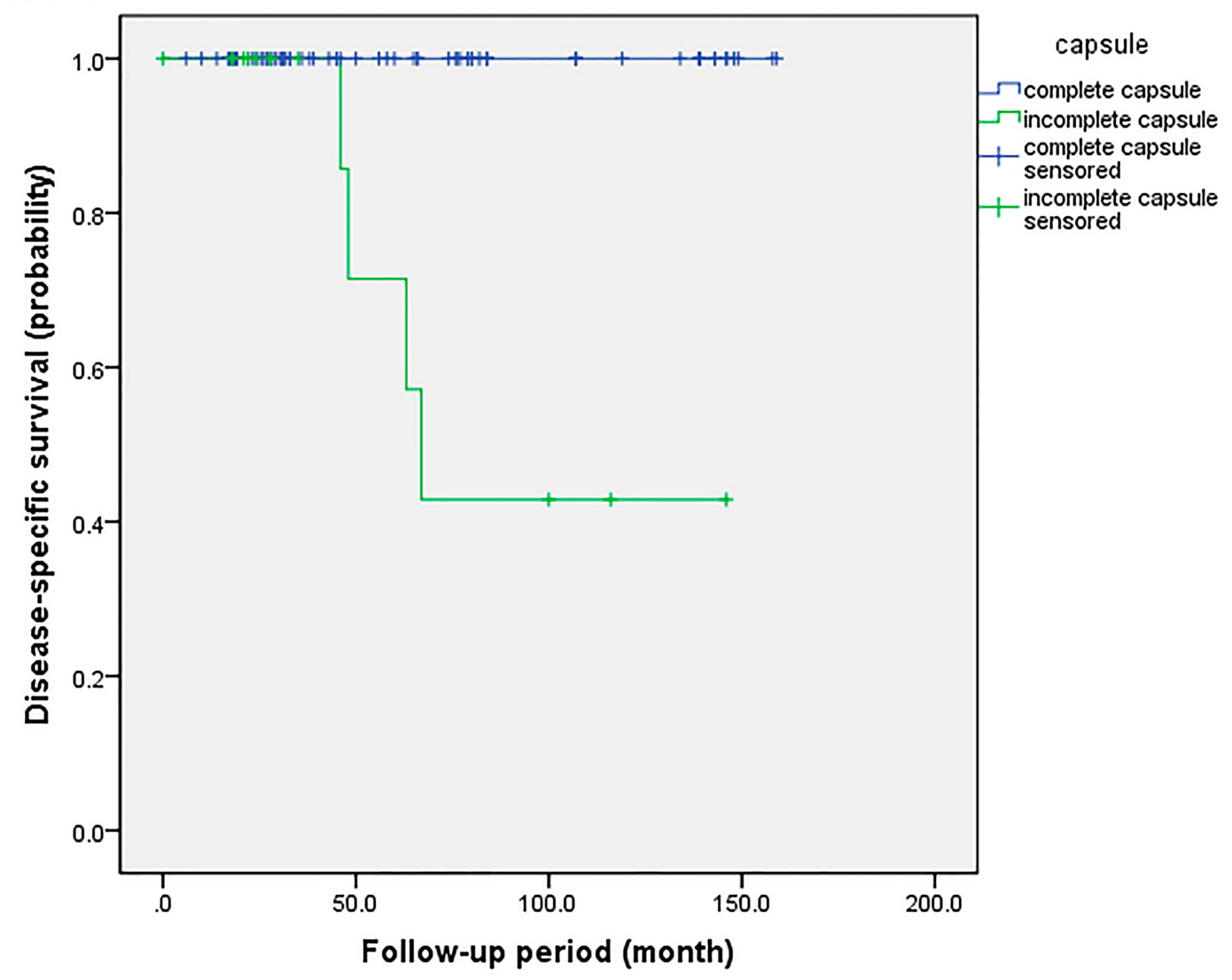

Figure 4 Cumulative Kaplan-Meier survival curve associated with capsule in SPTP. The patients with incomplete capsule had significantly shorter DSS than those with complete capsule.

peripancreatic fat tissue infiltration occurred in 8 cases, adjacent organ invasion was found in 6 cases, perineural invasion was present in 1 case. None had lymph node metastasis. Table 3 compares and summarizes the characteristics of patients with benign and malignant tumors. We found incomplete capsule $(p<0.001)$ and PNI $\leq 47.9(p=0.001)$ were presenting more significantly in the malignant group.

\section{Follow-Up Results}

Follow-up information was available for 97 patients with a median follow-up time of 59 months (6-159 months). Ninety-one patients were alive without evidence of disease at last follow-up, including 3 patients after resection of adjacent organs. Five cases of malignant SPTP (clinical details were shown in the supplemental file) suffered from recurrence or metastasis, and two underwent the second operation after 23 and 40 months of the first resection. Six patients died, 4 died of SPTP, 1 died of a traffic accident, and 1 died of heart failure. Five patients were lost to follow-up. The 3,5, and 10-year disease-specific survival was $100 \%, 97.9 \%$, and $95.8 \%$.
By univariate analysis, incomplete capsule $(p<0.001)$ and $\mathrm{PNI} \leq 47.9 \quad(p=0.013)$ were significant predictors of disease-specific survival (shown in Figures 4 and 5). Another two parameters with $p<0.1$, i.e. tumor size $(p=0.089)$ and component of tumor ( $p=0.081$ ) were also further conducted in the multivariate analysis. Incomplete capsule $(p=0.023)$ and $\mathrm{PNI} \leq 47.9(p=0.036)$ were still identified as the independent prognostic factor for DSS in SPTP patients. (shown in Table 4)

\section{Preoperative Prognostication of the coPNI-Capsule}

We established the prognostication combined PNI and capsule (coPNI-Capsule) in SPTP. Patients with complete capsule and high PNI were assessed as 2 points, patients with incomplete capsule and high PNI or with complete capsule and low PNI were assessed as 1 point, and patients with incomplete capsule and low PNI were assessed as 0 points. Three groups with scores of 0,1 , and 2 represent DSS with significant 


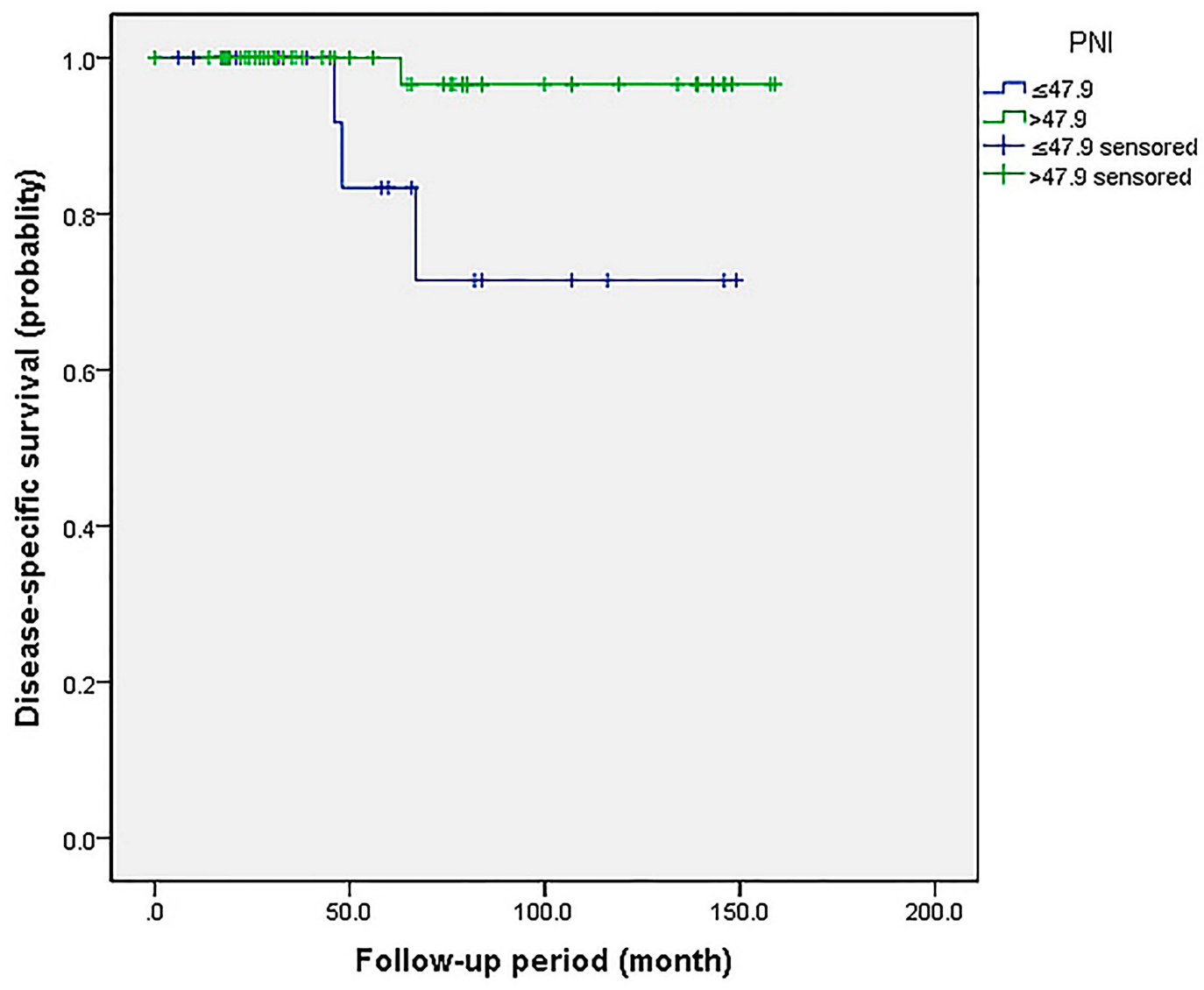

Figure 5 Cumulative Kaplan-Meier survival curve associated with PNI in SPTP. The patients with low PNI had significantly shorter DSS than those with high PNI.

difference $(p=0.001)$ (shown in Figure 6). The new stratification of coPNI-capsule could significantly predict the DSS in SPTP using the preoperative parameters.

\section{Discussion}

The low incidence of SPTP has postponed establishing a staging system for the management of patients. There is a paucity of standards for defining malignancy and prognosis by limited follow-up research. In recent years, the number of SPTP in China is growing; $\mathrm{Yu}$ et al and Pancreatic Surgery of Chinese Academic Society of Young, respectively, analyzed 553 cases and 713 cases in the Chinese population. ${ }^{13,14}$ Thus, due to the advantage of a large sample of Chinese patients, our study recruiting 102 cases during 14 years is convincing. To date, the largest sample SPTP of a single center in China is reported as 243 cases. $^{15}$

In our cohort, $17.6 \%$ (18/102) patients were diagnosed as malignant. Interestingly, in our study, none was observed for distant metastasis at initial diagnosis; however, three malignant cases suffered subsequent hepatic metastasis.

Previously many studies have illustrated the risk factors predicting malignancy. $\mathrm{Xu}$ et al found that the distal location of SPTP indicated malignancy, ${ }^{16}$ whereas other studies did not show any relation between location and malignancy. ${ }^{5,13,17} \mathrm{Ye}$ et al regarded that SPTP with incomplete capsule had larger tumor size compared to the complete capsule group. ${ }^{18}$ Song et al revealed that discontinuity capsule was not only the predictive factor of malignancy but also a significant predictor of diseasespecific survival. ${ }^{17}$ Meanwhile, Wang et al indicated that lack of complete capsule and age $>40.5$ years were independent risk factors of malignant SPTP. ${ }^{19} \mathrm{~A}$ metaanalysis involving articles in English revealed that malignant SPTP tended to be larger in diameter and younger in age than the benign type.$^{20}$ In recent years, the prognostic value of Ki-67 in SPTP obtained increasing attention. Some researchers considered that Ki-67 could be recommended as a valuable immunohistochemical parameter for predicting recurrence. ${ }^{21}$ In addition, 
Table 4 Prognostic Factors for DSS by Univariate and Multivariate Analysis

\begin{tabular}{|c|c|c|c|c|}
\hline \multirow[t]{3}{*}{ Variable } & \multicolumn{2}{|l|}{ Univariate Analysis } & \multicolumn{2}{|c|}{ Multivariate Analysis } \\
\hline & \multicolumn{2}{|l|}{ DSS } & \multicolumn{2}{|l|}{ DSS } \\
\hline & OR(95\% Cl) & $P$-value & OR(95\% Cl) & $P$-value \\
\hline \multicolumn{5}{|l|}{ Age } \\
\hline$<35$ & Reference & 0.267 & & \\
\hline$\geq 35$ & $0.535(0.325-1.716)$ & & & \\
\hline \multicolumn{5}{|l|}{ Gender } \\
\hline Female & Reference & 0.143 & & \\
\hline Male & $0.85 I(0.5 \mid 3-3.747)$ & & & \\
\hline \multicolumn{5}{|l|}{ BMI } \\
\hline$<18.5$ & Reference & 0.178 & & \\
\hline $18.5 \leq \leq 23.9$ & $4.347(0.277-16.574)$ & & & \\
\hline$>24$ & $2.013(0.062-7.865)$ & & & \\
\hline \multicolumn{5}{|l|}{ Symptoms } \\
\hline Present & Reference & 0.253 & & \\
\hline Absent & $4.655(0.689-7.231)$ & & & \\
\hline \multicolumn{5}{|l|}{ Tumor marker } \\
\hline Elevated & Reference & 0.455 & & \\
\hline Normal & $0.645(0.116-2.413)$ & & & \\
\hline \multicolumn{5}{|l|}{ Average tumor } \\
\hline$<5 \mathrm{~cm}$ & Reference & 0.089 & Reference & 0.321 \\
\hline$>5 \mathrm{~cm}$ & $1.067(0.93 \mathrm{I}-2.380)$ & & $\mathrm{I} .488(0.26 \mathrm{I}-2.639)$ & \\
\hline \multicolumn{5}{|l|}{ Calcification } \\
\hline Calcification & Reference & 0.731 & & \\
\hline Non-calcification & $2.323(0.013-8.165)$ & & & \\
\hline \multicolumn{5}{|l|}{ Tumor location } \\
\hline Head+neck & Reference & 0.121 & & \\
\hline Body and tail & $\mathrm{I} .65 \mathrm{I}(0.549-3.2 \mathrm{II})$ & & & \\
\hline Extrapancreatic site & $4.157(0.466-12.478)$ & & & \\
\hline \multicolumn{5}{|l|}{ Pattern of capsule } \\
\hline Complete capsule & Reference & 0.000 & Reference & 0.023 \\
\hline Incomplete capsule & $3.523(1.245-7.943)$ & & $1.218(1.161-2.639)$ & \\
\hline \multicolumn{5}{|l|}{ PNI } \\
\hline$>47.9$ & Reference & 0.013 & Reference & 0.036 \\
\hline$\leq 47.9$ & $2.454(I .42 I-5.152)$ & & $2.568(1.273-5.466)$ & \\
\hline \multicolumn{5}{|l|}{ Component } \\
\hline Cystic & Reference & $0.08 I$ & Reference & 0.434 \\
\hline Solid & $1.137(0.952-2.712)$ & & $3.950(0.563-8.231)$ & \\
\hline Solid and cystic & I.250(0.898-5.845) & & $4.089(0.021-|| .225)$ & \\
\hline
\end{tabular}

Ki-67 index $>4 \%$ was significantly related to poorer recurrence-free survival and disease-specific survival. ${ }^{5}$ The meta-analysis indicated that the risk of malignancy in SPTP became higher with the gradual increase of the Ki-67 index. ${ }^{22}$
Yang et al indicated that NLR might predict malignancy and recurrence-free survival. ${ }^{23}$ In our study, none of NLR, PLR, or SII were predictive of malignancy except for PNI. PNI, composed of albumin and lymphocytes, has been widely used not only as a marker of nutritional status 


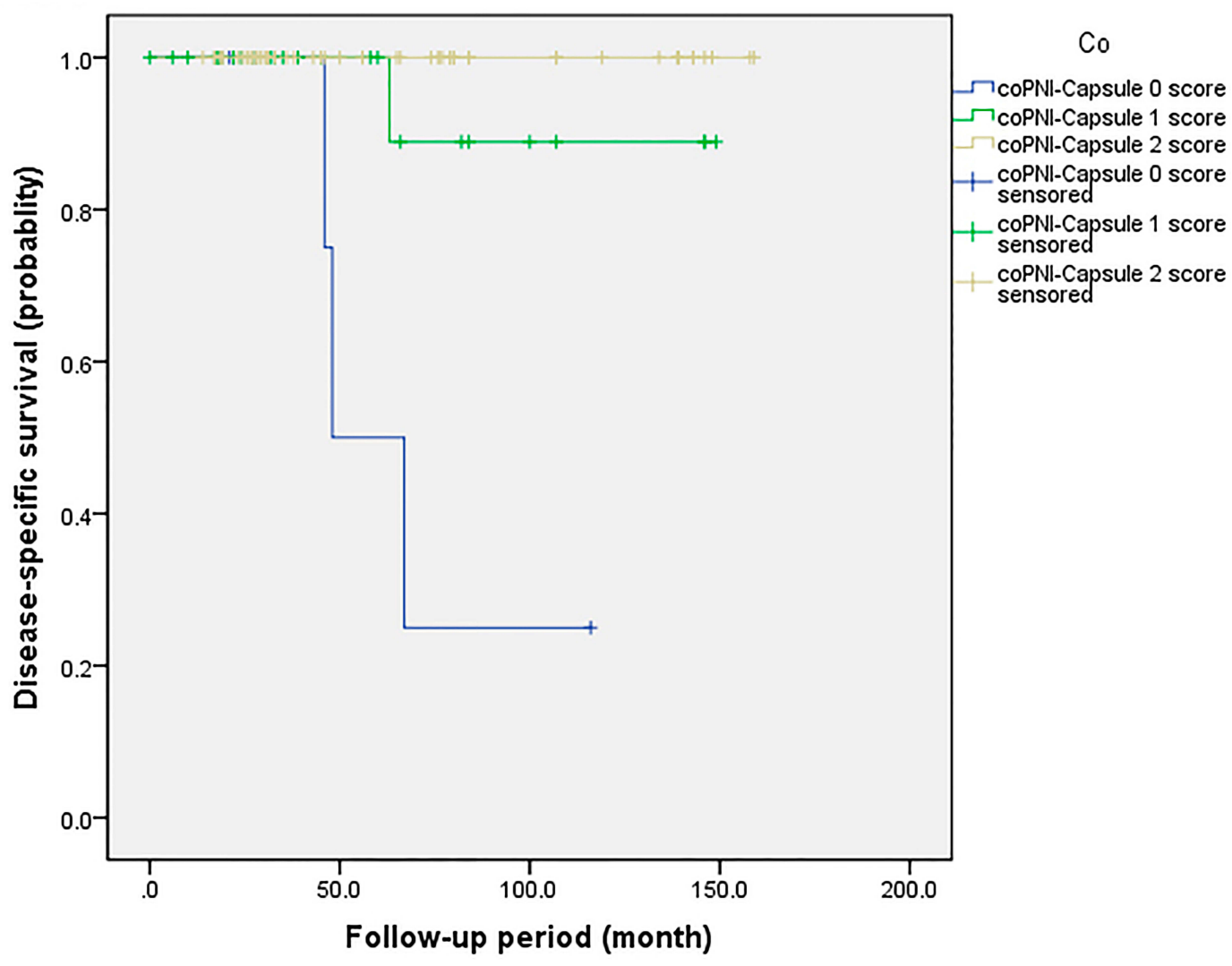

Figure 6 Cumulative Kaplan-Meier survival curve associated with coPNI-Capsule in SPTP. The patients with incomplete capsule and low PNI had significantly worse DSS.

but also a prognostic indicator in pancreatic cancer; ${ }^{8,24}$ however, there was no study focused on SPTP. To our knowledge, this is the first study to reveal PNI as a promising indicator for malignancy in SPTP. We defined the PNI as a binary variable as follows: PNI $\leq 47.9$; PNI $>47.9$. Then, we investigated that low PNI was significantly related to decreased DSS. Low PNI may be attributed to hypoalbuminemia and/or lymphocytopenia. Patients of pancreatic tumor evolving in protein malnutrition, probably suffered a decline in weight and albumin. ${ }^{25}$ On the other hand, lymphocytopenia might be an indication of a lower number of CD4+T helper lymphocytes, resulting in a poorer lymphocyte-mediated immune response to malignancies, contributing to tumor cell progression. $^{26}$

Imaging findings are also essential preoperative examination. SPTP often represented the mixed solid and cystic component with a well-circumscribed capsule. Chuang et al showed incomplete encapsulation was significantly observed in malignant SPTP, ${ }^{27}$ while the study did not detect the prognosis or survival for the further step. We found incomplete encapsulation to be a risk factor of malignancy and also a prognostic indicator of DSS.

The multivariate Cox analysis confirmed low PNI and incomplete capsule as independent prognostic factors. Therefore, we further combined PNI and capsule presence as the score 0,1 , and 2 to be evaluated. Three groups differ significantly in DSS. Patients with high PNI and complete capsule achieved the best prognosis, whereas patients with low PNI and incomplete capsule achieved the worst prognosis.

In clinical practice, the prognosis of SPTP is not based only on tumor resectable feasibility, but also takes into account the immunonutritional status of SPTP patients. Immunonutrition is thought to alter the production of cytokines and immune function. Hence, early nutritional intervention including oral immunonutrition is essential for the patients with low PNI preoperatively.

There are several limitations to our current investigation. Firstly, it is a single-center retrospective study. Secondly, the other well-known inflammatory indexes such as C-reactive protein, and the Glasgow score were not used for analysis, as a large portion of patients were 
not tested preoperatively. Finally, the AUC of determining PNI malignant is comparatively low. In spite of these limitations, this is the first study to reveal that PNI and incomplete capsule were reliable indicators of predicting malignancy and prognosis in SPTP. In future, a multicenter and large-sample study should be validated to corroborate our results.

\section{Conclusion}

In summary, our research confirmed that PNI value and capsule presence based on preoperative basic examination would be a simple and efficient tool to evaluate the natural history of SPTP. We suggest that patients with low $\mathrm{PNI} \leq 47.9$ and incomplete capsule should be monitored carefully and closely.

\section{Ethics}

This research was approved by the Ethics Committee of the First Hospital of China Medical University. Due to the retrospective study, patients' informed consent was waived. All research methods are in accordance with the regulations of the First Hospital of China Medical University. We declare that all the patient data are confidential and in compliance with the Declaration of Helsinki.

\section{Funding}

This work was supported in part by a grand-in aid for China Medical University Foundation for Young Key Teachers (No. 3110118149), Natural Science Foundation of Liaoning province (No. 2019-BS-282).

\section{Disclosure}

The authors declare that they have no conflicts of interest.

\section{References}

1. Papavramidis T, Papavramidis S. Solid pseudopapillary tumors of the pancreas: review of 718 patients reported in English literature. $J \mathrm{Am}$ Coll Surg. 2005;200(6):965-972. doi:10.1016/j.jamcollsurg.2005.02.011

2. Law JK, Ahmed A, Singh VK, et al. A systematic review of solidpseudopapillary neoplasms are these rare lesions? Pancreas. 2014;43 (3):331-337. doi:10.1097/MPA.0000000000000061

3. You L, Yang F, Fu D-L. Prediction of malignancy and adverse outcome of solid pseudopapillary tumor of the pancreas. World $J$ Gastrointest Oncol. 2018;10(7):184-193. doi:10.4251/wjgo.v10.i7.184

4. Kang CM, Choi SH, Kim SC, et al. Predicting recurrence of pancreatic solid pseudopapillary tumors after surgical resection: a multicenter analysis in Korea. Ann Surg. 2014;260(2):348-355. doi:10.1097/ SLA.0000000000000583

5. Yang F, Yu X, Bao Y, et al. Prognostic value of Ki-67 in solid pseudopapillary tumor of the pancreas: huashan experience and systematic review of the literature. Surgery. 2016;159(4):1023-1031. doi:10.1016/j.surg.2015.10.018
6. Shimizu T, Taniguchi K, Asakuma M, et al. Lymphocyte-to-monocyte ratio and prognostic nutritional index predict poor prognosis in patients on chemotherapy for unresectable pancreatic cancer. Anticancer Res. 2019;39(4):2169-2176. doi:10.21873/anticanres.13331

7. Ohno R, Kawamoto R, Kanamoto M, et al. Neutrophil to lymphocyte ratio is a predictive factor of malignant potential for intraductal papillary mucinous neoplasms of the pancreas. Biomark Insights. 2019;14:1177271919851505. doi:10.1177/1177271919851505

8. Nakagawa K, Sho M, Akahori T, et al. Significance of the inflammation-based prognostic score in recurrent pancreatic cancer. Pancreatology. 2019;19(5):722-728. doi:10.1016/j.pan.2019.05.461

9. Abe T, Nakata K, Kibe S, et al. Prognostic value of preoperative nutritional and immunological factors in patients with pancreatic ductal adenocarcinoma. Ann Surg Oncol. 2018;25(13):3996-4003. doi:10.1245/s10434-018-6761-6

10. Ganeshan DM, Paulson E, Tamm EP, Taggart MW, Balachandran A, Bhosale P. Solid pseudo-papillary tumors of the pancreas: current update. Abdom Imaging. 2013;38(6):1373-1382.

11. De Moura DTH, Coronel M, Ribeiro IB, et al. The importance of endoscopic ultrasound fine-needle aspiration in the diagnosis of solid pseudopapillary tumor of the pancreas: two case reports. J Med Case Rep. 2018;12(1):107. doi:10.1186/s13256-018-1585-3

12. Virgilio E, Mercantini P, Ferri M, et al. Is EUS-FNA of solid-pseudopapillary neoplasms of the pancreas as a preoperative procedure really necessary and free of acceptable risks? Pancreatology. 2014;14 (6):536-538. doi:10.1016/j.pan.2014.08.004

13. Yu P, Cheng X, Du Y, et al. Solid pseudopapillary neoplasms of the pancreas: a 19-year multicenter experience in China. $J$ Gastrointest Surg. 2015;19(8):1433-1440. doi:10.1007/s11605-015-2862-8

14. Pancreatic Surgery of Chinese Academic Society of Young, S. The current status of diagnosis and treatment of pancreatic cystic neoplasm in China: a report of 2251 cases. Zhonghua Wai Ke Za Zhi. 2018;56(1):24-29. doi:10.3760/cma.j.issn.0529-5815.2018.01.007

15. Liu M, Liu J, Hu Q, et al. Management of solid pseudopapillary neoplasms of pancreas: a single center experience of 243 consecutive patients. Pancreatology. 2019;19(5):681-685. doi:10.1016/j.pan.20 19.07.001

16. Xu Y, Zhao G, Pu N, et al. One hundred twenty-one resected solid pseudopapillary tumors of the pancreas: an 8-year single-institution experience at Zhongshan Hospital, Shanghai, China. Pancreas. 2017;46(8):1023-1028. doi:10.1097/MPA.0000000000000885

17. Song H, Dong M, Zhou J, et al. Solid pseudopapillary neoplasm of the pancreas: clinicopathologic feature, risk factors of malignancy, and survival analysis of 53 cases from a single center. Biomed Res Int. 2017;2017:5465261. doi:10.1155/2017/5465261

18. Ye J, Ma M, Cheng D, et al. Solid-pseudopapillary tumor of the pancreas: clinical features, pathological characteristics, and origin. $J$ Surg Oncol. 2012;106(6):728-735. doi:10.1002/jso.23195

19. Wang J, Chen X, Wang C, et al. Differentiation of aggressive from non-aggressive pancreatic solid pseudopapillary neoplasms using computed tomography. Abdom Radiol. 2019;44(7):2448-2458. doi:10.1007/s00261-019-01969-6

20. Song H, Dong M, Xiao H, et al. A meta-analysis: could we predict the malignancy of solid pseudopapillary neoplasm? Int J Clin Exp Pathol. 2017;10(9):9113-9121.

21. Zou C, Yang F, Fu D. Meta-analysis of Ki-67 expression for recurrence in patients with solid pseudopapillary tumor of the pancreas. HPB (Oxford). 2020;22(4):631-632.

22. Zou C, Yang F, Wu W, et al. Ki-67 and malignancy in solid pseudopapillary tumor of the pancreas: a systematic review and meta-analysis. Pancreatology. 2020;20(4):683-685. doi:10.1016/j.pan.2020. 03.015

23. Yang F, Bao Y, Zhou Z, et al. Preoperative neutrophil-to-lymphocyte ratio predicts malignancy and recurrence-free survival of solid pseudopapillary tumor of the pancreas. J Surg Oncol. 2019;120(2):241248. doi:10.1002/jso.25484 
24. Ichikawa I, Mizuno S, Hayasaki A, et al. Prognostic nutritional index after chemoradiotherapy was the strongest prognostic predictor among biological and conditional factors in localized pancreatic ductal adenocarcinoma patients. Cancers. 2019;11(4):19. doi:10.33 90/cancers 11040514

25. Todorov P, Cariuk P, McDevitt T, et al. Characterization of a cancer cachectic factor. Nature. 1996;379(6567):739-742. doi:10.1038/ 379739a0
26. De Giorgi U, Mego M, Scarpi E, et al. Relationship between lymphocytopenia and circulating tumor cells as prognostic factors for overall survival in metastatic breast cancer. Clin Breast Cancer. 2012;12(4):264-269. doi:10.1016/j.clbc.2012.04.004

27. Chung YE, Kim M-J, Choi J-Y, et al. Differentiation of benign and malignant solid pseudopapillary neoplasms of the pancreas. J Comput Assist Tomogr. 2009;33(5):689-694. doi:10.1097/RCT.0b013e318 $18 \mathrm{f} 2 \mathrm{a} 74$

\section{Publish your work in this journal}

Cancer Management and Research is an international, peer-reviewed open access journal focusing on cancer research and the optimal use of preventative and integrated treatment interventions to achieve improved outcomes, enhanced survival and quality of life for the cancer patient
The manuscript management system is completely online and includes a very quick and fair peer-review system, which is all easy to use. Visit http://www.dovepress.com/testimonials.php to read real quotes from published authors. 